\title{
Towards a Theory of Instantons at Non-zero Fermion Density
}

\author{
Gregory W. Carter ${ }^{a}$ and Dmitri Diakonov ${ }^{b}$ \\ ${ }^{a}$ Niels Bohr Institute, Blegdamsvej 17, 2100 Copenhagen Ø, Denmark

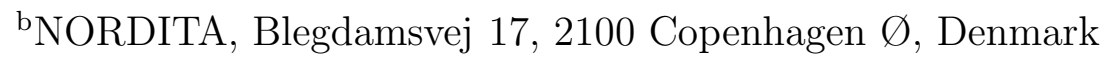

A formalism to study light fermions at non-zero chemical potential in the instanton medium is presented. It is then applied to investigate chiral symmetry restoration and diquark condensation at finite baryon density.

\section{INTRODUCTION}

The fact that instanton-induced interactions produce attraction not only in the $\bar{q} q$ channel (leading to the spontaneous chiral symmetry breaking) but also in the $q q$ channel (potentially leading to diquark condensation) has been first realized in ref. [1]. It becomes especially clear when the number of colours is $N_{c}=2$, where instanton-induced interactions possess a global $S U(4)$ symmetry [2] (often referred to as Pauli-Gürsey symmetry). For this reason $\bar{q} q$ and $q q$ condensates belong in fact to one phase: one condensate can be rotated to another along the Goldstone valley [2].

The possibility of diquark condensation (in analogy to superconductivity) at any $N_{c}$, as induced by instantons, has been studied in ref. [3]. Only a metastable diquark-condensed vacuum has been found at $N_{c}>2$ and chemical potential $\mu=0$. Furthermore, already at $N_{c}=3$ the scalar diquark appears to be unbound in the vacuum, indicating that our world is in a sense closer to the idealized $N_{c} \rightarrow \infty$ than to the $N_{c}=2$ world. Parametrically, the diquark mass is $\sim 1 / \bar{\rho} \sim 1 \mathrm{GeV}$ where $\bar{\rho}$ is the average instanton size as explained below. It means that a scalar diquark correlation function should decay with the exponent corresponding to the 'constituent' quark threshold $2 M(0) \approx 700 \mathrm{MeV}$, which seems to

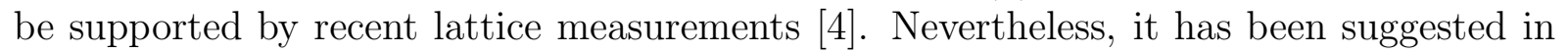
[3] that $q q$ condensates could be found as metastable states in heavy ion collisions and in astrophysics.

Quite recently diquark condensation owing to instantons at $\mu \neq 0$ has been estimated by the Stony Brook and Princeton/MIT groups [5,6], see also these proceedings. Below we present a formalism to study it in a more systematic way. A theory of light quarks in the instanton vacuum, though not completely trivial, is rather well developed, and it is straightforward to generalize it to $\mu \neq 0$. We will avoid some of the unnecessary approximations made in [5, 6]. 


\section{QCD INSTANTON VACUUM}

The Euclidean QCD partition function for $N_{f}$ fermions with a common chemical potential $\mu$ is

$$
\mathcal{Z}=\int D A_{\mu} \exp \left(-\int \frac{F_{\mu \nu}^{a 2}}{4 g^{2}}\right) \int D \psi D \psi^{\dagger} \exp \left[\sum_{f} \int \psi_{f}^{\dagger}\left(i \not \nabla+i m_{f}-i \mu \gamma_{4}\right) \psi_{f}\right]
$$

It is understood that the theory is regularized in some way at large momenta, for example, by the Pauli-Villars method. It is also convenient to normalize the partition function to the free one. The integral over fermion fields can be thus understood as

$$
\int D \psi D \psi^{\dagger} \exp [\ldots] \equiv \operatorname{Det}=\prod_{f} \frac{\operatorname{det}\left(i \not \nabla+i m_{f}-i \mu \gamma_{4}\right) \operatorname{det}(i \not \partial+i M)}{\operatorname{det}(i \not \nabla+i M) \operatorname{det}\left(i \not \partial+i m_{f}\right)}
$$

where $M$ is the Pauli-Villars regulator mass playing the role of the ultraviolet cutoff.

The main hypothesis of the instanton vacuum is that the partition function (11) is mainly saturated by the ensemble of instantons and antiinstantons (I's and $\bar{I}$ 's for short), plus quantum fluctuations about them. Since the ensemble of $I$ 's and $\bar{I}$ 's is not, strictly speaking, a solution of the Yang-Mills equation of motion (it is only in the infinitely dilute limit), one has to do something more clever than the saddle-point integration over $A_{\mu}$. The most straightforward procedure is to use the Feynman variational principle in order to get the lower bound for the partition function (1) [7]. Writing the general Yang-Mills field as a superposition of $N_{+} I$ 's and $N_{-} \bar{I}$ 's plus a presumably weak quantum field $B_{\mu}$,

$$
A_{\mu}=\sum A_{\mu}^{I}(\xi)+\sum A_{\mu}^{\bar{I}}(\xi)+B_{\mu}
$$

where $\xi$ is a set of $4 N_{c}$ collective coordinates for each instanton (namely center, size and colour orientation), one can show that the partition function (11) can be approximated by

$$
\mathcal{Z} \geq \sum \frac{1}{N_{+} ! N_{-} !} \int d \xi J(\xi) \exp \left[-U_{\text {int }}(\xi)\right] \cdot \text { Det. }
$$

Here $J$ is a Jacobian which is due to passing to the collective coordinates and $U_{\text {int }}$ is the instanton interaction. Actually, the separation of the dependence on the collective coordinates $\xi$ into $J$ and $U_{\text {int }}$ is ansatz-dependent since the precise meaning of the collective coordinates depends on the functional form of the field. However, their combination should be ansatz-independent, at least if one calculates both quantities with infinite precision. We shall assume the simplest sum ansatz (3).

It has been shown in ref. [7] (see also a more recent paper [8]) that the partition function (4) (without the fermion determinant) leads to the stabilization of the grand canonical instanton ensemble. The basic characteristics of the medium is the average instanton size, $\bar{\rho}$, and the average separation between near neighbours, $\bar{R}$, or the instanton density at equilibrium, $N / V=1 /(\bar{R})^{4}$. Since the transmutation of dimensions takes place in this approach, both quantities are expressed through the only dimensional parameter one has in the pure glue theory, namely $\Lambda_{Q C D}$. The 2-loop calculations performed in [7,8] give

$$
\bar{\rho}=\sqrt{\overline{\rho^{2}}} \approx \frac{0.48}{\Lambda_{\overline{M S}}}, \quad \bar{R} \approx \frac{1.35}{\Lambda_{\overline{M S}}}
$$


Taking $\Lambda_{\overline{M S}}=280 \mathrm{MeV}$ one finds $\bar{\rho} \approx 0.35 \mathrm{fm}, \quad \bar{R} \approx 0.95 \mathrm{fm}, \bar{\rho} / \bar{R} \approx 1 / 3$. This small ratio has been previously suggested on phenomenological grounds by Shuryak [9]. The smallness of the $\bar{\rho} / \bar{R}$ ratio implies that the packing fraction of instantons in the vacuum, i.e. the fraction of the $4 \mathrm{~d}$ volume occupied by the balls of radius $\bar{\rho}$, is quite small:

$$
f=\frac{\pi^{2}}{2} \bar{\rho}^{4} \frac{N}{V}=\frac{\pi^{2}}{2} \frac{\bar{\rho}^{4}}{\bar{R}^{4}} \sim \frac{1}{10} .
$$

\subsection{Instanton Packing Fraction as Algebraic Parameter}

After the transmutation of dimensions takes place all physical quantities are proportional to $\Lambda_{Q C D}$ in appropriate powers with numerical coefficients of the order of unity, as it should be, generally speaking, in a strong interaction theory. Therefore, if one gets a numerically small (or large) dimensionless quantity it must be due to some exceptional reason. In our case the reason why one gets a numerically small packing fraction (6) can be traced back to the 'accidentally' large value $11 / 3$ of the coefficient in the asymptotic freedom law. The same analysis applied to the $2 \mathrm{~d} C P^{N}$ model also possessing instantons shows that there are no such numerical tricks there: instantons of that model seem to be strongly overlapping, and there appears no small packing fraction parameter.

Though $\bar{\rho} / \bar{R} \approx 1 / 3$ is but numerically small, one can treat it as a formal algebraic parameter, and develop a perturbation theory in it. In principle, one can change the parameter $\bar{\rho} / \bar{R}$ by taking more quark flavours, or taking nonzero temperature or including Higgs field which cuts off integrals over the instanton sizes.

\subsection{Separating High and Low Fermion Eigenmodes}

We now switch on $N_{f}$ flavours of light quarks represented by the fermion determinant (2) in the backround field of the instanton ensemble. The crucial feature of fermions in the one-instanton background is that there is an exact zero mode [10. In the infinitely dilute limit of instantons that would lead to the nullification of the fermion determinant. Though the packing fraction of instantons is small it is, however, nonzero, and one can build a systematic theory of light quarks in the instanton vacuum by treating $\bar{\rho} / \bar{R}$ as a formal small parameter 1113 .

An important step is to separate contributions of high- and low-frequency parts to the fermion determinant. The role of the high-frequency part is to renormalize somewhat the one-instanton weight or fugacity. The low-frequency part is physically much more interesting: it is responsible for chiral symmetry breaking in the vacuum and its restoration at high densities and/or temperatures.

The separation is made in respect to an auxiliary mass $M_{1}$ lying inside a parametrically wide interval, $1 / \bar{R} \ll M_{1} \ll 1 / \bar{\rho}$. One rewrites the fermion determinant (国) as a product,

$$
\text { Det }=\frac{\operatorname{det}\left(i \not \nabla+i m_{f}-i \mu \gamma_{4}\right) \operatorname{det}(i \not \partial+i M)}{\operatorname{det}(i \not \nabla+i M) \operatorname{det}\left(i \not \partial+i m_{f}\right)}=\operatorname{Det}_{H i g h} \cdot \operatorname{Det}_{L o w},
$$

where

$$
\operatorname{Det}_{H i g h}=\frac{\operatorname{det}\left(i \not \nabla+i M_{1}\right) \operatorname{det}(i \not \partial+i M)}{\operatorname{det}(i \not \nabla+i M) \operatorname{det}\left(i \not \partial+i M_{1}\right)}, \quad \operatorname{Det}_{L o w}=\frac{\operatorname{det}\left(i \not \nabla+i m_{f}-i \mu \gamma_{4}\right) \operatorname{det}\left(i \not \partial+i M_{1}\right)}{\operatorname{det}\left(i \not \nabla+i M_{1}\right) \operatorname{det}\left(i \not \partial+i m_{f}\right)}
$$

These ratios are arranged so that Det $_{\text {High }}$ gets a contribution from fermion modes with Dirac eigenvalues ranging from $M_{1}$ to the Pauli-Villars mass $M$, while Det $_{\text {Low }}$ is saturated 
by eigenvalues less than $M_{1}$. The product of the two determinants is, by construction, independent of the separation scale $M_{1}$. However, we are going to treat both of them approximately. The fact that the actual dependence of the product on $M_{1}$ turns out to be extremely feeble [11] in the wide range of $M_{1}$ serves as a check of the approximations.

In considering Det $\operatorname{High}_{\text {, }}$ one assumes that $M_{1}$ is large enough so that $\operatorname{Det}_{\text {High }}$ can be factorized into a product of determinants computed in the background of individual instantons. Corrections to the factorization are of the order of $\left(M_{1} \bar{R}\right)^{-2} \ll 1$.

In considering $\operatorname{Det}_{\text {Low }}$, one assumes that $M_{1}$ is small enough so that only the (diagonalized) would-be zero modes of individual instantons can be taken into account. Corrections from nonzero modes of individual instantons are of the order of $\left(M_{1} \bar{\rho}\right)^{2} \ll 1$.

Taking nonzero chemical potential $\mu$ does not seriously change the above logic. At $\mu \bar{\rho} \ll 1$ (which will be our domain of interest since all important phenomena happen inside this range) the $\mu$ term should be included into the 'low' part as in eq. (7).

\subsection{Three Ways to See Chiral Symmetry Breaking}

In refs.111 13 three seemingly different but actually equivalent ways of computing Det $_{\text {Low }}$ have been developed, thus leading to several languages in which chiral symmetry breaking by instantons can be understood and mathematically described.

The first method is based upon diagonalizing the overlaps of the would-be zero fermion modes of individual instantons [11, 12]. One can write

$$
\operatorname{Det}_{L o w}=\exp \left\{\frac{1}{2} \int d \lambda \nu(\lambda) \ln \frac{\lambda^{2}+m^{2}}{\lambda^{2}+M_{1}^{2}}\right\}
$$

where $\nu(\lambda)$ is the spectral density of the Dirac operator in the field of the instanton ensemble. It is obtained from diagonalizing a matrix made of the overlap integrals of the would-be zero modes, and then averaging over the ensemble. The nonzero $\nu(0)$ signals chiral symmetry breaking: it is actually due to the delocalization of the zero modes owing to the "hopping" of quarks from one instanton to another.

The second method is to find the quark propagator in the instanton ensemble. In the zero-mode approximation (justified when the packing fraction (6) is small) one can write a closed equation for the quark propagator averaged over the instanton ensemble [12,14]. The propagator appears to be that of a massive fermion with a momentumdependent mass $M(p)$ related to the Fourier transform of the zero mode. Using the main characterictics of the instanton vacuum (5) one gets $M(0) \approx 350 \mathrm{MeV}$ which is what one expects for the so-called constituent quark mass. Parametrically, $M(0) \sim \sqrt{N / V N_{c}} \bar{\rho}$, meaning that the constituent quark mass is small in the packing fraction of instantons.

Integrating the quark propagator over the 4-momentum one gets the chiral condensate $\langle\bar{\psi} \psi\rangle \approx-(250 \mathrm{MeV})^{3}$ being also close to its phenomenological value.

The $\operatorname{Det}_{\text {Low }}$ can be expressed through the propagator $S(x, y)$ as

$$
\operatorname{Det}_{\text {Low }}=\exp \int_{m}^{M_{1}} i d m^{\prime} \operatorname{Tr}\left[S\left(x, x, m^{\prime}\right)-S_{0}\left(x, x, m^{\prime}\right)\right]
$$

where $S_{0}$ is the free propagator. Comparing the integrand in eq. (9) with that of eq. (8) one finds the Wigner semicircle spectral density of the Dirac operator averaged over the instanton ensemble [12, which can also be obtained directly from the first method 15]. 
Both methods can be directly generalized to nonzero chemical potential, since at $\mu \neq 0$ there also exists an exact normalizable zero fermion mode 16,17. However, in this paper we use the third method suggested in [13] which is also easily translated to $\mu \neq 0$.

\section{EFFECTIVE FERMION ACTION AT $\mu \neq 0$}

Rather than calculating fermionic quantities in the background of instantons and then averaging over these configurations, one can perform the averaging procedure first. This generates instanton-induced interactions in the form of a vertex involving $2 N_{f}$ quarks. It is a direct consequence of the appearance of the quark zero modes, one for each flavour, and the resulting interactions are naturally of the 't Hooft form [10].

The guiding principle is to write a fermion partition function reproducing the propagator at low momenta as well as the fermion determinant. This derivation has been developed in detail in previous publications [13] and its extension to finite chemical potential is straightforward. It should be stressed that correlations between instantons induced by fermions are inherent in this approach; as to correlations induced by gluons, they are effectively taken care of by the use of the variational principle [0] resulting in the effective size distribution. For simplicity we freeze all the sizes at the average value $\bar{\rho}$, but average explicitly over randomly positioned and oriented instantons.

The partition function which produces the necessary propagator is of the form

$$
\mathcal{Z}=\operatorname{Det}_{L o w}=\int D \psi D \psi^{\dagger} \exp \left[\sum_{f} \int \psi_{f}^{\dagger}\left(i \not \partial-i \mu \gamma_{4}\right) \psi_{f}\right]\left(\frac{Y_{N_{f}}^{+}}{V}\right)^{N_{+}}\left(\frac{Y_{N_{f}}^{-}}{V}\right)^{N_{-}}
$$

The pre-exponential factors contain the instanton-induced interactions between fermions, and for $N_{f}$ flavours are the specific nonlocal $2 N_{f}$-fermion vertices

$$
Y_{N_{f}}^{ \pm}=(-)^{N_{f}} \int d^{4} z d U \prod^{N_{f}} \int d^{4} x d^{4} y \psi_{L, R}^{\dagger}(x)(i \partial-i \mu)^{\mp} \Phi_{\bar{I}, I}(x-z) \tilde{\Phi}_{\bar{I}, I}(y-z)(i \partial-i \mu)^{ \pm} \psi_{L, R}(y) .(11
$$

Here we use the notation $x^{ \pm}=x^{\mu} \sigma_{\mu}^{ \pm}$, where the $2 \times 2$ matricies $\sigma_{\mu}^{ \pm}=( \pm i \vec{\sigma}, 1)$ decompose the Dirac matricies into chiral components, and it is understood that $\mu$ written as a fourvector is $\mu_{\alpha}=(\overrightarrow{0}, \mu)$. Note that the zero mode in the field of an (anti) instanton couples to that of a (right-) left-handed quark. By $\tilde{\Phi}_{I(\bar{I})}$ we denote the conjugate zero mode. As noticed in [16], $\tilde{\Phi}_{I}(x, \mu)=\Phi_{I}^{\dagger}(x,-\mu)$, where the dagger means hermitean conjugate.

Fermion operators in the pre-exponent are not convenient; these operators can be raised into the exponent with the help of a supplementary integration over a pair of Lagrange multipliers, denoted $\lambda_{ \pm}$:

$$
\begin{aligned}
\mathcal{Z}=\int d \lambda_{+} d \lambda_{-} & \int D \psi D \psi^{\dagger} \exp \left\{\int d^{4} x \psi^{\dagger}\left(i \not \partial-i \mu \gamma_{4}\right) \psi+\lambda_{+} Y_{N_{f}}^{+}+\lambda_{-} Y_{N_{f}}^{-}\right. \\
& \left.+N_{+}\left(\ln \frac{N_{+}}{\lambda_{+} V}-1\right)+N_{-}\left(\ln \frac{N_{-}}{\lambda_{-} V}-1\right)\right\}
\end{aligned}
$$

Indeed, integrating over $\lambda_{ \pm}$by the saddle-point method one recovers eq. (10) (the saddlepoint integration becomes exact in the thermodynamic limit $\left.N_{ \pm} \rightarrow \infty\right)$. Through this procedure we obtain a purely exponential integrand which is the required effective action. 
It is very important that the overall strength of the $2 N_{f}$-fermion interaction, whose role is played by $\lambda_{ \pm}$, is not fixed once and forever: actually its value is found from minimizing the free energy after integration over fermions is performed. Therefore the strength of the interaction depends itself on the phase the fermion system assumes. As will be shown below, this is the mechanism by which chiral symmetry is restored at large chemical potential.

Although one can investigate the phenomena of chiral symmetry breaking and its restoration for any number of flavours, we shall restrict ourselves to the case $N_{f}=2$ corresponding to a system of chiral up and down quarks. Furthermore, we will assume the CP invariant case of $\theta=0$, which requires $N_{+}=N_{-}=N / 2$ and hence $\lambda_{+}=\lambda_{-}=\lambda_{\text {. }}$ For practical applications it is favourable to use the Fourier-transformed expressions of the quark zero modes, which are written explicitly in the Appendix. These complex functions of the four-momenta and chemical potential determine the (matrix) form factors attached to each fermion leg of the vertex:

$$
\mathcal{F}(p, \mu)=(p+i \mu)^{-} \varphi(p, \mu)^{+}, \quad \mathcal{F}^{\dagger}(p,-\mu)=\varphi^{*}(p,-\mu)^{-}(p+i \mu)^{+} .
$$

With such definitions, the interaction terms may be written in momentum space,

$$
\begin{aligned}
\lambda_{+} Y^{+}=\lambda_{+} & \int \frac{d^{4} p_{1} d^{4} p_{2} d^{4} k_{1} d^{4} k_{2}}{(2 \pi)^{16}}(2 \pi)^{4} \delta^{4}\left(p_{1}+p_{2}-k_{1}-k_{2}\right) \\
& \cdot \int d U \psi_{L 1 \alpha_{1} i_{1}}^{\dagger}\left(p_{1}\right) \mathcal{F}\left(p_{1}, \mu\right)_{k_{1}}^{i_{1}} \epsilon^{k_{1} l_{1}} U_{l_{1}}^{\alpha_{1}} U_{\beta_{1}}^{\dagger o_{1}} \epsilon_{n_{1} o_{1}} \mathcal{F}^{\dagger}\left(k_{1},-\mu\right)_{p_{1}}^{n_{1}} \psi_{L}^{1 \beta_{1} p_{1}}\left(k_{1}\right) \\
& \cdot \psi_{L 2 \alpha_{2} i_{2}}^{\dagger}\left(p_{2}\right) \mathcal{F}\left(p_{2}, \mu\right)_{k_{2}}^{i_{2}} \epsilon^{k_{2} l_{2}} U_{l_{2}}^{\alpha_{2}} U_{\beta_{2}}^{\dagger o_{2}} \epsilon_{n_{2} o_{2}} \mathcal{F}^{\dagger}\left(k_{2},-\mu\right)_{p_{2}}^{n_{2}} \psi_{L}^{2 \beta_{2} p_{2}}\left(k_{2}\right),
\end{aligned}
$$

with a similar form for $\lambda_{-} Y^{-}$which carries right-handed quarks. The first indices on the fermion operators refer to flavour (1 or 2 explicitly), the Greek to colour $\left(1 \ldots N_{c}\right)$, and the last denote spin $(1,2)$. This formulation of the effective interaction retains the full $p$ and $\mu$ dependence of the zero modes, as opposed to the approximated treatments in other recent works [5, 6].

\section{COMPETITION BETWEEN $\bar{q} q$ AND $q q$ CHANNELS}

Since the instanton-induced interactions (14) support both $\bar{q} q$ and $q q$ condensation, it is necessary to consider the two competing channels simultaneously. This means that one must calculate both the normal $(G)$ and anomalous $(F)$ quark Green functions. A colour/flavour/spin ansatz compatible with the possibility of chiral and colour symmetry breaking is

$$
\begin{aligned}
\left\langle\psi^{f \alpha i}(p) \psi_{g \beta j}^{\dagger}(p)\right\rangle & =\delta_{g}^{f} \delta_{\beta}^{\alpha} S_{1}(p)_{j}^{i} \quad \text { for } \alpha, \beta=1,2, \\
\left\langle\psi^{f \alpha i}(p) \psi_{g \beta j}^{\dagger}(p)\right\rangle & =\delta_{g}^{f} \delta_{\beta}^{\alpha} S_{2}(p)_{j}^{i} \quad \text { for } \alpha, \beta>2, \\
\left\langle\psi_{L}^{f \alpha i}(p) \psi_{L}^{g \beta j}(-p)\right\rangle & =\left\langle\psi_{R}^{f \alpha i}(p) \psi_{R}^{g \beta j}(-p)\right\rangle=\epsilon^{f g} \epsilon^{\alpha \beta[\gamma]} \epsilon^{i j} F(p),
\end{aligned}
$$

where $[\gamma]$ refers to some generalized direction(s) in colour space, and it is this set of $N_{c}-2$ indices which signals the breaking of colour symmetry. In the particular case of $N_{c}=3$, where the colour symmetry is broken as $S U(3) \rightarrow S U(2) \times U(1)$, we will by convention take $[\gamma]=3$; for $N_{c}=4$ one can take $[\gamma]=34$ and so forth. In the event of colour 


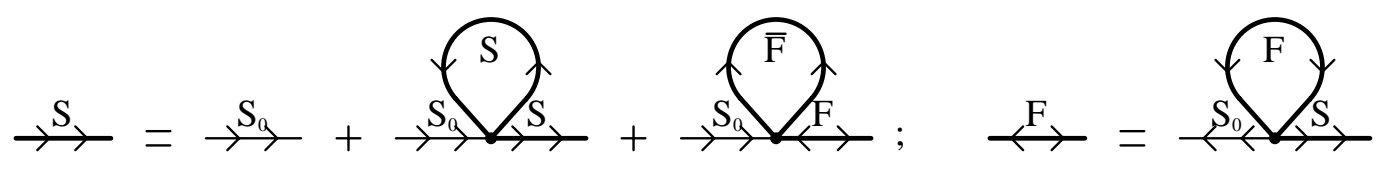

Figure 1. Gorkov equations to first order in $\lambda$.

symmetry breaking, the standard propagators (and ensuing condensates) will lose their colour degeneracy and the separation of $S(p)$ into $S_{1}(p)$ and $S_{2}(p)$ becomes necessary; otherwise the Schwinger-Dyson equations do not close.

Written in the chiral $L, R$ basis, the $4 \times 4$ propagators $S_{1,2}(p)$ are of the form:

$$
S(p)=\left[\begin{array}{cc}
G(p) \mathbf{1} & Z(p) \mathbf{S}_{0}(p)^{+} \\
Z(p) \mathbf{S}_{0}(p)^{-} & G(p) \mathbf{1}
\end{array}\right], \quad \mathbf{S}_{0}(p)^{ \pm}=\left[(p+i \mu)^{ \pm}\right]^{-1}
$$

Here the off-diagonal, bare propagator is modified by the scalar functions $Z_{1,2}(p)$, and is augmented on the diagonal by the scalar $G_{1,2}(p)$ which if nonzero break chiral symmetry.

Using the instanton-induced interaction (14) one can build a systematic expansion for the $F, G$ Green functions in the $1 / N_{c}$ and $\bar{\rho} / \bar{R}$ parameters. In the leading order in both parameters we restrict ourselves to the one-loop approximation shown in Fig. 11. It corresponds to a set of self-consistent Schwinger-Dyson equations (called Gorkov equations in the case of superconductivity with $F \neq 0$ ). An important $\mu$-dependence enters through the form factors in eq. (14). With $\bar{F}(p)=F^{*}(p)$ these diagrams lead to the set of five algebraic equations for the scalar Green functions $Z_{1,2}, G_{1,2}$ and $F$ :

$$
\begin{aligned}
Z_{1}(p) & =1-G_{1}(p) A(p, \mu) \bar{g}_{1}-F(p) B(p, \mu) \bar{f} \\
Z_{2}(p) & =1-G_{2}(p) A(p, \mu) \bar{g}_{2} \\
G_{1}(p) & =Z_{1}(p) \varphi_{\alpha}(p, \mu) \varphi_{\alpha}(p, \mu) \bar{g}_{1} \\
G_{2}(p) & =Z_{2}(p) \varphi_{\alpha}(p, \mu) \varphi_{\alpha}(p, \mu) \bar{g}_{2} \\
F(p) & =Z(-p) \varphi_{\alpha}(p, \mu) \varphi_{\alpha}(-p, \mu) \bar{f}
\end{aligned}
$$

The numerical factors $\bar{g}_{1}, \bar{g}_{2}$, and $\bar{f}$ will be defined below and the functions

$$
\begin{aligned}
A(p, \mu)= & (p+i \mu)_{\alpha}(p+i \mu)_{\alpha} \varphi_{\beta}(p, \mu) \varphi_{\beta}(p, \mu) \\
B(p, \mu)= & \left(p^{2}+\mu^{2}\right) \varphi_{\beta}(p, \mu) \varphi_{\beta}(-p, \mu)+(p+i \mu)_{\alpha} \varphi_{\alpha}(p, \mu)(p-i \mu)_{\beta} \varphi_{\beta}(-p, \mu) \\
& \quad-(p+i \mu)_{\alpha} \varphi_{\alpha}(-p, \mu)(p-i \mu)_{\beta} \varphi_{\beta}(p, \mu)
\end{aligned}
$$

are the form factors which arise from the zero modes (see the Appendix). At $\mu=0$ we have $A(p, 0)=B(p, 0)$, but for any finite $\mu$ the direction of the momentum flow through each vertex leg is critical.

The condensates $g_{1}, g_{2}$, and $f$ are the closed loops contributing to the quark self-energy. They are found by integrating the appropriate Green function, modified by the vertex form factors, over an independent momentum:

$$
g_{1,2}=\frac{\lambda}{N_{c}^{2}-1} \int \frac{d^{4} k}{(2 \pi)^{4}} A(k, \mu) G_{1,2}(k), \quad f=\frac{\lambda}{N_{c}^{2}-1} \int \frac{d^{4} k}{(2 \pi)^{4}} B(k, \mu) F(k) .
$$


These appear in the particular combinations

$$
\begin{aligned}
\bar{g}_{1} & =\left(5-\frac{4}{N_{c}}\right) g_{1}+\left(2 N_{c}-5+\frac{2}{N_{c}}\right) g_{2}, \quad \bar{g}_{2}=2\left(2-\frac{1}{N_{c}}\right) g_{1}+2\left(N_{c}-2\right) g_{2}, \\
\bar{f} & =2\left(1+\frac{1}{N_{c}}\right) f .
\end{aligned}
$$

Although the integrands above are complex, all imaginary parts are odd in $p_{4}$ and thus vanish under integration. As the $\bar{g}_{1,2}$ are measures of chiral symmetry breaking, these act as an effective mass modifying the standard quark propagation. On the other hand the diquark loop $\bar{f}$ plays a different role, that of twice the single-quark energy gap (conventionally denoted $\Delta$ ) formed around the Fermi surface. The Fermi momentum, in the absence of chiral symmetry breaking, will remain at $p_{f}=\mu$ regardless of the magnitude of $\bar{f}$.

After determining the five scalar functions through solving eqs. (17) and inserting these solutions into eqs. (19) we find the coupled equations for the condensates themselves:

$$
\begin{aligned}
g_{1} & =\frac{\lambda \bar{g}_{1}}{N_{c}^{2}-1} \int \frac{d^{4} k}{(2 \pi)^{4}} \frac{\alpha\left(1-\beta \bar{f}^{2}+\alpha^{*} \bar{g}_{1}^{2}\right)}{\left(1+\alpha \bar{g}_{1}^{2}\right)\left(1+\alpha^{*} \bar{g}_{1}^{2}\right)-\beta^{2} \bar{f}^{4}}, \quad g_{2}=\frac{\lambda \bar{g}_{2}}{N_{c}^{2}-1} \int \frac{d^{4} k}{(2 \pi)^{4}} \frac{\alpha}{1+\alpha \bar{g}_{2}^{2}}, \\
f & =\frac{\lambda \bar{f}}{N_{c}^{2}-1} \int \frac{d^{4} k}{(2 \pi)^{4}} \frac{\beta\left(1-\beta \bar{f}^{2}+\alpha \bar{g}_{1}^{2}\right)}{\left(1+\alpha \bar{g}_{1}^{2}\right)\left(1+\alpha^{*} \bar{g}_{1}^{2}\right)-\beta^{2} \bar{f}^{4}},
\end{aligned}
$$

where yet another pair of functions has been introduced,

$$
\alpha(p, \mu)=A(p, \mu) \varphi_{\alpha}(p, \mu) \varphi_{\alpha}(p, \mu), \quad \beta(p, \mu)=B(p, \mu) \varphi_{\alpha}(p, \mu) \varphi_{\alpha}(-p, \mu) .
$$

Note that while $\beta$ is real, the funtion $\alpha$ is complex. As usual for gap equations, there is the possibility of a solution where some (or even all) of the condensates vanish.

The magnitudes of these condensates are not yet determined, as this requires fixing the coupling constant $\lambda$. This is done through minimizing the partition function (12) and leads to

$$
\frac{N}{V}=\lambda\left\langle Y^{+}+Y^{-}\right\rangle
$$

On the left-hand side is the instanton density, which we will here take to be fixed at its vacuum value, although in principle will have some correction due to the finite quark density. Evaluating the right-hand side requires calculating the one-vertex contributions to the free energy, which in this case includes two-loop, figure-eight type diagrams formed by joining the four fermion legs into two pairs as shown in Fig. 2. This can be written concisely in terms of the condensates:

$$
\frac{N}{V}=\lambda\left\langle Y^{+}+Y^{-}\right\rangle=\frac{4\left(N_{c}^{2}-1\right)}{\lambda}\left[2 g_{1} \bar{g}_{1}+\left(N_{c}-2\right) g_{2} \bar{g}_{2}+2 f \bar{f}\right] .
$$

This equation for $\lambda$ and the definitions of the condensates (21) comprise a system of equations which can be solved for all quantities.

Once this has been achieved the chiral condensate may be calculated, which is a closed trace over the quark propagator. This is distinct from the condensates $\bar{g}_{1,2}$, however it 


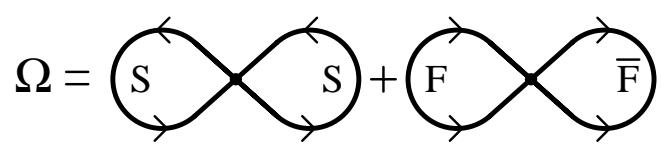

Figure 2. Contributing diagrams to $\Omega$ at order $\lambda^{1}$.

does contain the same properties under $p_{4}$ reflection which guarantee it is real. It is most simply written

$$
-\langle\bar{\psi} \psi\rangle_{\text {Mink }}=i\left\langle\psi^{\dagger} \psi\right\rangle_{E u c l}=i \int \frac{d^{4} p}{(2 \pi)^{4}} \operatorname{Tr} S(p)=4 \int \frac{d^{4} p}{(2 \pi)^{4}}\left[2 G_{1}(p)+\left(N_{c}-2\right) G_{2}(p)\right] .
$$

As a consequence of having two different modes of quark condensation, one obtains multiple solutions for the characteristics of the quark medium at any fixed chemical potential. Specifically, there will be a competition between the following phases:

(0) No condensation of any type; $g_{1}=g_{2}=f=0$.

(1) Chiral symmetry breaking without diquark condensation. This, the standard vacuum scenario, has the signature $g_{1}=g_{2} \neq 0, f=0$.

(2) Diquark condensation without chiral breaking, or $f \neq 0, g_{1}=g_{2}=0$.

(3) A mixed phase wherein both symmetries are broken, and $g_{1}, g_{2}$, and $f$ are all distinct and finite.

Phase (0) requires that $\lambda$ vanish, and such a solution was never found in our calculations. To resolve between the remaining, symmetry-breaking phases, the free energy is minimized. Consistent with the evaluation of the Green functions, it is calculated to first order in $\lambda$. Repeating the calculation of figure-eight diagrams and recalling the explicit dependence on $\lambda$ in eq. (12) we obtain the free energy

$$
\frac{\Omega}{V_{3}}=-\frac{1}{\beta V_{3}} \ln Z=\frac{\Omega_{0}}{V_{3}}-\frac{N}{V} \ln \left(\frac{N}{\lambda V}\right)+\frac{N}{V}-\frac{4\left(N_{c}^{2}-1\right)}{\lambda}\left[2 g_{1} \bar{g}_{1}+\left(N_{c}-2\right) g_{2} \bar{g}_{2}+2 f \bar{f}\right] .
$$

Here $V_{3}$ is the three-volume while $V$ is the Euclidean four-volume, and $\Omega_{0}$ is the free energy for a gas of free quarks. The last two terms are precisely the quantity which must vanish under the saddle-point determination of $\lambda$, and thus we have

$$
\frac{\Omega}{V_{3}}=\frac{\Omega_{0}}{V_{3}}+\frac{N}{V} \ln \left(\frac{\lambda}{N / V}\right) \text {. }
$$

Thus the phase which features the lowest coupling $\lambda$ is the thermodynamically favoured.

Numerically, no solutions were found which favour phase (3), the mixed case. Thus we concentrate on the distinction between phases (1) and (2), the former being marked by nonzero $g=g_{1}=g_{2}$, the latter by nonzero $f$. Each case leads to its own value of $\lambda$ through solving eq. (24) for any given $\mu$. At certain value of $\mu=\mu_{c}$ the solutions for $\lambda$ corresponding to the two phases cross; this is the point where the phase transition occurs. This point is defined by the condition

$$
\frac{f\left(\mu_{c}\right)}{g\left(\mu_{c}\right)}=\sqrt{\frac{N_{c}\left(N_{c}-1\right)}{2}} .
$$

When the ratio between the condensates is less than the constant on the right, phase (2) is favoured; otherwise it is chiral symmetry that is spontaneously broken in phase (1). Calculations were carried out for various $N_{c}$, taking the values for $N / V$ and $\bar{\rho}$ specified in Section 2. 


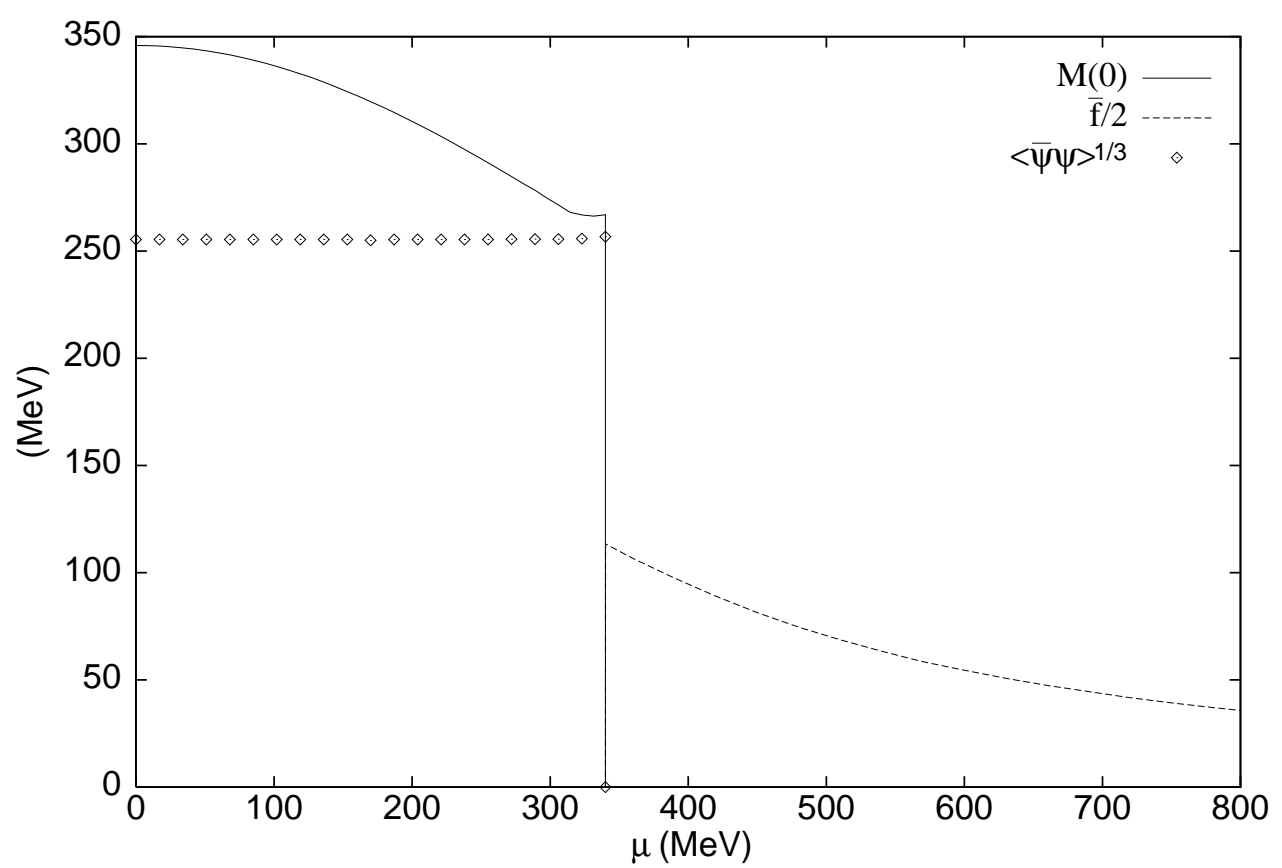

Figure 3. Condensates for $N_{c}=3$ as a function of $\mu$. Shown are the effective quark mass $M(0)$, the quark condensate $\langle\bar{\psi} \psi\rangle^{1 / 3}$, and the diquark energy gap per quark $\bar{f} / 2$.

\subsection{The Case of $N_{c}=2$}

In this case it is obvious that colour symmetry is not broken by diquark formation, which here correspond to colour-singlet 'baryons', and hence there is only one possible chiral condensate $g_{1}=g$. Not so obvious is that at $\mu=0$ the colour and flavour $S U(2)$ groups are arranged into the higher $S U(4)$ symmetry. The instanton vacuum accounts for this symmetry [2], and in the context of the analysis here this corresponds to $f^{2}+g^{2}$ being the only discernable quantity in the gap equations (21). Numerically, we find $\sqrt{f^{2}+g^{2}}=147 \mathrm{MeV}$.

At finite $\mu$, however, the $S U(4)$ symmetry is explicity broken and one finds $f<g$. Since in this case the critical ratio of eq. (28) is unity, we conclude that for any finite density the $N_{c}=2$ world prefers diquark condensation to chiral symmetry breaking. This finding is in agreement with the reasoning of ref. [5] and the lattice results of ref. [18].

\subsection{The Case of $N_{c}=3$}

For three colours, the critical ratio of the condensates (28) is $\sqrt{3}$. At $\mu=0$ we find $f / g=$ $165 \mathrm{MeV} / 65 \mathrm{MeV}>\sqrt{3}$. It means that at low values of $\mu$ the coupling constant $\lambda$ is smaller in the chiral broken phase, hence this phase is energetically preferred. Furthermore, the effective quark mass is the reasonable $M(0)=\bar{g}=346 \mathrm{MeV}$. We thus recover the standard chiral symmetry breaking at low baryon density.

With increasing chemical potential both $f$ and $g$ condensates in their respective phases are reduced, however as $\mu$ surpasses the effective quark mass $M(0)$ around $300 \mathrm{MeV}$, the chiral-breaking $g$ stabilizes while the colour-breaking $f$ continues to decrease in phase (2). At $\mu \simeq 340 \mathrm{MeV}$ condition (28) is reached, and therefore for this and greater chemical potential chiral symmetry is restored and the 'colour superconducting' phase is realized. At the transition point, the quark gap is $\bar{f} / 2=115 \mathrm{MeV}$ and slowly decreasing with rising 
density. Numerical results for the characteristic quantities of each phase are plotted in Fig. 3. It is noteworthy that the chiral condensate, $\langle\bar{\psi} \psi\rangle$, is distinct from the effective quark mass, $\bar{g}$, in that while the latter decreases with $\mu$ the former remains practically at its vacuum value. For $\mu>\mu_{c}$ they both vanish.

\section{CONCLUSIONS}

We have formulated the effective low-energy fermion action induced by instantons at nonzero chemical potential. In the resulting fermion vertex we have retained the full dependence on both momentum and chemical potential, which arise from the would-be zero modes. In this respect we differ from other studies such as the random matrix model, NJL models, and alternative instanton approaches.

The effective action leads to a competition between two phases, one of chiral symmetry breaking and another characterized by diquark condensation. It was studied by solving a coupled system of gap equations to first order in the instanton density. For two massless flavours and three colours, it was found that the usual broken chiral symmetry is restored through a first order phase transition, replaced by colour breaking due to the formation of a diquark condensate. This occurs at a critical chemical potential of $\simeq 340 \mathrm{MeV}$.

\section{APPENDIX. Fourier Transforms of Fermion Zero Modes}

The use of the exact fermion zero modes in the momentum space tremendously simplifies all calculations. The starting point is the exact fermion zero mode in the field of one (anti)instanton in $x$ space [16,17], which we cite for arbitrary instanton position $z$, size $\rho$ and orientation given by rectangular $N_{c} \times 2$ matrix $U$ :

$$
\begin{aligned}
& {[\Phi(x-z)]_{i}^{\alpha}=\frac{\rho}{\sqrt{2} \pi} e^{\mu\left(x_{4}-z_{4}\right)} \sqrt{\Pi(x-z)}\left[\not \partial\left(\frac{e^{-\mu\left(x_{4}-z_{4}\right)} \Delta(x-z, \mu)}{\Pi(x-z)}\right) \frac{1 \pm \gamma_{5}}{2}\right]_{i j} \epsilon^{j k} U_{k}^{\alpha} .} \\
& \Pi(x)=1+\frac{\rho^{2}}{x_{4}^{2}+r^{2}+\rho^{2}}, \quad \Delta(x, \mu)=\frac{1}{x_{4}^{2}+r^{2}}\left[\cos (\mu r)+\frac{x_{4}}{r} \sin (\mu r)\right]
\end{aligned}
$$

Its Fourier transform is defined as $\Phi(p, \mu)=i \int \mathrm{d}^{4} x e^{-i p \cdot x} \Phi(x, \mu)$ and has the structure $\Phi(p, \mu)=\gamma_{\alpha} \varphi_{\alpha}(p, \mu)$. The Lorentz symmetry is broken at finite $\mu$, and the components become

$$
\begin{aligned}
\varphi_{4}\left(p_{4}, p ; \mu\right)=\frac{\pi \rho^{2}}{4 p}\{( & \left(p-\mu-i p_{4}\right)\left[\left(2 p_{4}+i \mu\right) f_{1-}+i\left(p-\mu-i p_{4}\right) f_{2-}\right] \\
+ & \left.\left(p+\mu+i p_{4}\right)\left[\left(2 p_{4}+i \mu\right) f_{1+}-i\left(p+\mu+i p_{4}\right) f_{2+}\right]\right\} \\
\varphi_{i}\left(p_{4}, p ; \mu\right)=\frac{\pi \rho^{2} p_{i}}{4 p^{2}}\{( & (2 p-\mu)\left(p-\mu-i p_{4}\right) f_{1-}+(2 p+\mu)\left(p+\mu+i p_{4}\right) f_{1+} \\
+ & {\left[2(p-\mu)\left(p-\mu-i p_{4}\right)-\frac{1}{p}\left(\mu+i p_{4}\right)\left[p_{4}^{2}+(p-\mu)^{2}\right]\right] f_{2-} } \\
+ & {\left[2(p+\mu)\left(p+\mu+i p_{4}\right)+\frac{1}{p}\left(\mu+i p_{4}\right)\left[p_{4}^{2}+(p+\mu)^{2}\right]\right] f_{2+}(31) }
\end{aligned}
$$


where the scalar $p=|\vec{p}|$, the spatial $i=1 \ldots 3$, and the functions

$$
f_{1 \pm}=\frac{I_{1}\left(z_{ \pm}\right) K_{0}\left(z_{ \pm}\right)-I_{0}\left(z_{ \pm}\right) K_{1}\left(z_{ \pm}\right)}{z_{ \pm}}, \quad f_{2 \pm}=\frac{I_{1}\left(z_{ \pm}\right) K_{1}\left(z_{ \pm}\right)}{z_{ \pm}^{2}}
$$

are evaluated at $z_{ \pm}=\frac{1}{2} \rho \sqrt{p_{4}^{2}+(p \pm \mu)^{2}}$. With these expressions it is explicitly verified that the normalization condition holds for any $\mu$ :

$$
1=\int \frac{d^{4} p}{(2 \pi)^{4}} \tilde{\Phi}_{I}(p, \mu) \Phi_{I}(p, \mu)=\int \frac{d^{4} p}{(2 \pi)^{4}}\left[\varphi_{4}^{*}(-\mu) \varphi_{4}(\mu)+\vec{\varphi}^{*}(-\mu) \cdot \vec{\varphi}(\mu)\right]
$$

\section{REFERENCES}

1. R.G.Betman and L.V.Laperashvili, Sov. J. Nucl. Phys. 41 (1985) 295.

2. D.Diakonov and V.Petrov, Diquarks in the Instanton Picture, in: Quark Cluster Dynamics, Lecture Notes in Physics, Eds. K.Goeke, P.Kroll and H.Petry, Springer-Verlag (1992) p.288.

3. D.Diakonov, H.Forkel and M.Lutz, Phys. Lett. B373 (1996) 147.

4. M.Hess, F.Karsch, E.Lärmann and I.Wetzorke, Diquark Masses in Lattice QCD, heplat/9804023.

5. R.Rapp, T.Schäfer, E.V.Shuryak and M.Velkovsky, Diquark Bose Condensates in High Density Matter and Instantons, hep-ph/9711396.

6. M.Alford, K.Rajagopal, and F.Wilczek, Phys. Lett. B422 (1998) 247.

7. D.Diakonov and V.Petrov, Nucl. Phys. B245 (1984) 259.

8. D.Diakonov, M.Polyakov and C.Weiss, Nucl. Phys. B461 (1996) 539.

9. E.Shuryak, Nucl. Phys. B203 (1982) 93.

10. G.'t Hooft, Phys. Rev. D14 (1976) 3432; Erratum: ibid. D18 (1978) 2199.

11. D.Diakonov and V.Petrov, Phys. Lett. 147B (1984) 351.

12. D.Diakonov and V.Petrov, Sov. Phys. JETP 62 (1985) 204, 431;

Nucl. Phys. B272 (1986) 457.

13. D.Diakonov and V.Petrov, Spontaneous Breaking of Chiral Symmetry in the Instanton Vacuum, preprint LNPI-1153 (1986), published (in Russian) in: Hadron Matter under Extreme Conditions, Kiev (1986) p.192, see also D.Diakonov, hep-ph/9602375, hepph/9802298.

14. P.Pobylitsa, Phys. Lett. 226B (1989) 387.

15. Yu.A.Simonov, Phys. Rev. D43 (1991) 3534.

16. A.A.Abrikosov Jr., Nucl. Phys. B182 (1981) 441; Sov. J. Nucl. Phys. 37 (1983) 459.

17. C.A. de Carvalho, Nucl. Phys. B183 (1981) 182.

18. E. Dagotto, F. Karsch and A. Moreo, Phys. Lett. 169B (1986) 421. 